\title{
Foster Relative
}

National Cancer Institute

\section{Source}

National Cancer Institute. Foster Relative. NCI Thesaurus. Code C71393.

Unrelated persons who are part of the same foster family. 